\title{
Research on the Lack of School Identity Caused by the Impact of Class Teaching on the Self-esteem of Ordinary Students
}

\author{
Wang Ziyu \\ International College, Xiamen University, Xiamen, Fujian361000, China \\ *Email:1592446117@qq.com
}

\begin{abstract}
Exploring the lack of school identity caused by the impact of class teaching on the self-esteem of ordinary class students, this paper uses the method of questionnaire research, based on the class teaching questionnaire, two-dimensional self-esteem scale, and school identity questionnaire, a total of 821 students from grade 1 to grade 2 of ordinary class in some middle schools in Jiangxi, Hubei, and Anhui provinces. Results: $\square$ class teaching was positively correlated with school identity, and had a significant positive predictive effect on school identity. Self-esteem plays a partial mediating role between adolescent school identity and class teaching. $\square$ The self-esteem of male and female students has some mediating effect between class teaching and school identity, but the effect of mediating effect of female students $(51.2 \%)$ is greater than that of male students $(39.8 \%)$. Conclusion: class teaching can attack the self-esteem of ordinary students and lead to the lack of school identity, and the influence effect of girls is greater.
\end{abstract}

Keywords: class teaching, School identity, self-esteem, sex difference, education fair.

\section{THE INTRODUCTION}

\subsection{Teaching in Different Classes}

The teaching method of divided classes is derived from the hierarchical teaching method[1]. Stratified teaching method refers to the teaching method that students are divided into different groups or classes based on grades, knowledge mastery, and learning ability, and teachers carry out targeted teaching for students of different levels. However, the class-dividing teaching principle is the explicit manifestation of the class-dividing teaching method, which is generally adopted in China to divide the school classes into "key classes" and "ordinary classes" according to the differences in students' scores. Ordinary class, also known as a parallel class, generally refers to the class composed of students ranked by the entrance examination results of the middle and lower, equipped with ordinary teacher resources; the key class, also known as the top class or experimental class, is composed of students with excellent scores in the entrance examination and equipped with excellent teacher resources[2].

For many years, class placement is controversial, a foreign scholars named Francis (2017) put forward the question about teaching method, thinking that the essence of "hierarchy" is "students"[3], domestic scholar hong-yan cheng (2009), the combination of Slavin banding theory and the field survey results point KuaiManBan teaching can improve students' grades, will also have psychological pressure on student[4]. Most scholars hold the view that classifying students according to their grades will harm those with poor grades. Students who are assigned to "poor classes" will have a stereotype of "poor students", which will strike the self-esteem of these students and bring about many negative psychological effects. The school's training goal is to make students get moral, intellectual, physical, aesthetic, labor, and all-around development, not only to improve students' academic performance but also to pay attention to students' mental health problems. Studies have shown that "the mental health of students in regular classes is generally worse than that of students in key classes"[5]. The purpose of this paper is to study the impact of class teaching on the self-esteem of ordinary class students and their school identity. 


\subsection{Recognition by the school}

"Identity" is a concept of great concern in the field of humanities, especially psychology and sociology. Specifically, it refers to an individual's value-oriented judgment and evaluation of himself and his surroundings. School identity refers to students' positive spiritual experience and positive behavior of the school, which includes students' cognition, emotion, motivation, and behavior of the school.

Many school ideological educators believe that school identity is an indispensable cornerstone of school education. Whether students can cultivate their identity directly affects the construction of school spirit to a certain extent, thus affecting the overall and healthy development of the school[6]

There are three factors influencing students' school identity: students themselves, the school itself, and the external environment or social environment. This paper focuses on the individual factors of students[7], the sense of school support obtained by students, that is, the campus resources and development opportunities obtained by students during the school, and explores the impact of class teaching on students' self-esteem and their sense of school support, to further produce the impact on school identity.

\subsection{Self-esteem}

The concept of self-esteem first appeared in the late 19th century. Mruk (2013) believes that self-esteem is a living phenomenon unique to human beings and refers to the ability of individuals to constantly deal with life challenges in a valuable way. The earliest self-esteem research officially entered the field of psychology is American psychologist James, in his book the psychology principle, self-esteem is introduced into the system itself, in the process of the discussion of the "self" concept, also has carried on the detailed instructions for self-esteem, believe that self-esteem is based on self-consciousness and self-action. Self-esteem is thought to be closely related to one's achievements and high aspirations; It's also thought to be an experience of an individual's sense of efficacy. Related studies have also found that high self-esteem can bring not only positive effects but also corresponding negative effects[8]. As a dynamic system of individual psychological activities, self-esteem is the main driving force[9] of group identity affecting individual psychological and social behaviors and plays an important mediating role[10]. Therefore, the self-esteem of ordinary class students may also play a mediating role in the relationship between class placement and school identity development.

To sum up, this study will explore the relationship between class placement and school identity, as well as the mediating role of self-esteem and gender differences[11].

\section{RESEARCH METHODS}

\subsection{Research Objects}

Some students from grade one to grade two in six middle schools in Wuhan, Hefei, Shiyan, and Wuyuan were selected as questionnaire research objects. A total of 900 questionnaires were distributed by online sampling, and 821 were effectively received with an effective recovery rate of $91.2 \%$. There were 368 boys and 453 girls. There are 190 students in grade one, 182 students in Grade two, 157 students in Grade three, 120 students in grade one, and 172 students in grade two.

\subsection{Research Tools}

\subsubsection{Opinion questionnaire on class-based teaching method}

By searching the data, we made a questionnaire on the opinion of the teaching method of the class. The questionnaire has 10 questions, including the opinion of the teaching method of the class, the effect of the teaching method of the class, and the influence of the teaching method of the class. The likert5-point scoring method is adopted, $1=$ very inconsistent, $5=$ very consistent. The higher the score is, the higher the students' recognition of the teaching method.

\subsubsection{School Identification Questionnaire}

It is excerpted from the college student identification questionnaire prepared by Ding Li. The questionnaire has 40 questions, including the four dimensions of group cognition, emotional connection, positive evaluation, and independent behavior, using the likert5-point scoring method, $1=$ very inconsistent, 5= very consistent. The higher the score, the higher the student's school identification.

\subsubsection{Self-esteem questionnaire}

The self-esteem scale was originally developed by Rosenberg in 1965 to assess adolescents' overall feelings about self-worth and self-acceptance. The scale consists of 10 items, including 5 positive scores and 5 negative scores. The subjects reported directly whether these descriptions matched themselves[12]. It is rated on a scale of four, with 1 being very consistent, 2 being consistent, 3 being inconsistent and 4 being very inconsistent. The overall score ranges from 10 to 40 , with higher scores associated with higher self-esteem.

\subsubsection{Data analysis}

SPSS was used for data entry, management, and 
correlation analysis, and $\mathrm{M}$ plus was used for structural equation model analysis of mediation.

\subsection{Result Analysis}

\subsubsection{Test of common method deviation}

To check the problem of common method bias, this study conducted the Haman single-factor test and found that in the case of no rotation, the eigenvalues of 13 factors were greater than 1 , and the explanation rate of the first factor was $23.54 \%$, less than the critical standard of $40 \%$, indicating that the effect of common method bias was not prominent.

\subsubsection{Correlation analysis between variables}

There is a significant positive correlation between class classification, student self-esteem, and school identity. In addition, the correlation between gender and self-esteem was significant (see Table 1).

\subsubsection{Mediating role of self-esteem}

To investigate the relationship between school identity and class placement and the mediating effect of self-esteem. In this study, MPLus7.0 is used to construct and verify the structural equation model. In the model analysis, the result variable was class-based teaching, the school identity was the predictive variable, and the student self-esteem was the intermediary variable. Among them, class teaching is a significant variable, while school identity and self-esteem are latent variables.

The results of the analysis of the model with the whole data showed that the fitting of all indicators of the whole model reached the acceptable standard, $\chi 2 / \mathrm{DF}=$ 5.80,TLI $=0.94, \mathrm{CFI}=0.97, \mathrm{RMSEA}=0.08, \mathrm{SRMR}=0.045$, and the normalized path coefficients were shown in Figure 1. The path coefficient structure chart shows that class teaching has a significant direct positive prediction effect on self-esteem and school identity of ordinary class students $(\beta=0.338, \mathrm{P}<0.01 ; \beta=0.218, \mathrm{P}<0.01)$; The positive effect of self-esteem on school identity was also significant $(\beta=0.492, \quad \mathrm{P}<0.01)$. This indicated that self-esteem had a partial mediating effect between class placement and school identity, and the mediating effect (effect value $=0.166, \mathrm{P}<0.01 ; 95 \%$ confidence interval: $0.112-0.223$ ) accounted for $43.27 \%$ of the total effect.

Table 1 Descriptive statistics and correlation analysis among variables $(\mathrm{N}=861)$

\begin{tabular}{|c|c|c|c|c|c|c|c|c|c|c|c|}
\hline & $\mathrm{M}$ & SD & 1 & 2 & 3 & 4 & 5 & 6 & 7 & 8 & 9 \\
\hline 1 gender & - & - & - & & & & & & & & \\
\hline 2Divide into classes teaching & 3.72 & 0.62 & 0.03 & - & & & & & & & \\
\hline 3Ability to evaluate & 3.46 & 0.65 & -0.01 & $0.68^{* *}$ & - & & & & & & \\
\hline 4Learning attitude & 3.83 & 0.81 & 0.04 & $0.87^{* *}$ & $0.51^{* *}$ & - & & & & & \\
\hline 5Self assessment & 3.76 & 0.76 & -0.01 & $0.91^{* *}$ & $0.49^{* *}$ & $0.75^{\star *}$ & - & & & & \\
\hline 6Mentality change & 3.79 & 0.69 & $0.08^{*}$ & $0.86^{* *}$ & $0.45^{\star *}$ & $0.66^{* *}$ & $0.69^{* *}$ & - & & & \\
\hline 7self-esteem & 3.11 & 0.62 & $-0.16^{\star *}$ & $0.35^{\star *}$ & $0.50^{* *}$ & $0.35^{\star *}$ & $0.26^{\star *}$ & $0.31^{* *}$ & - & & \\
\hline 8Group identification & 3.26 & 0.73 & $-0.15^{\star *}$ & $0.34^{* *}$ & $0.25^{\star *}$ & $0.34^{* *}$ & $0.26^{\star *}$ & $0.30^{* *}$ & $0.94^{* *}$ & - & \\
\hline 9Emotional connection & 2.97 & 0.61 & $-0.14^{\star *}$ & $0.30^{* *}$ & $0.24^{* *}$ & $0.30^{* *}$ & $0.22^{* *}$ & $0.27^{* *}$ & $0.91^{* *}$ & $0.70^{* *}$ & - \\
\hline 10 School identity & 2.94 & 0.75 & -0.01 & $0.38^{* *}$ & $0.30^{* *}$ & $0.32^{* *}$ & $0.26^{\star *}$ & $0.40^{* *}$ & $0.90^{* *}$ & $0.38^{* *}$ & $0.55^{\star *}$ \\
\hline
\end{tabular}

Note: Male is 0 , female is $1 ; * \mathrm{P}<0.05, \mathrm{P}<0.01$. * *

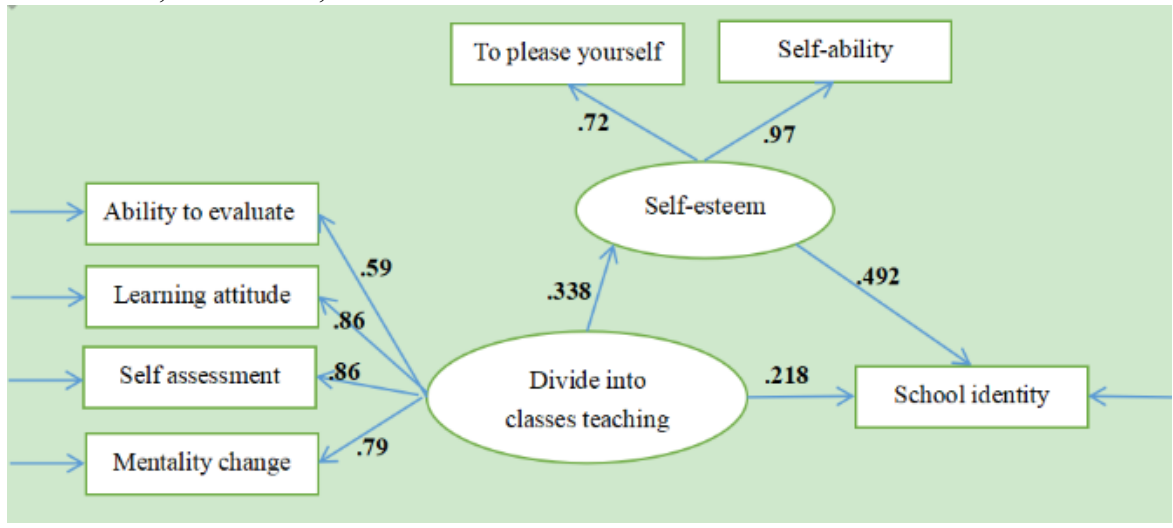

Note: $* * \mathrm{P}<0.01$

FIG. 1 Mediating effect of self-esteem

\subsubsection{Gender difference in mediating effect of self-esteem}

To determine whether there is a gender difference in the mediating effect of self-esteem, two group models of boys and girls were established according to the same method above[13 14]. The results showed that all 
fitting indexes of male and female models were in an acceptable range (see Table 2).

Table 2 Fitting parameters of male and female models

\begin{tabular}{|l|l|l|l|l|l|}
\hline & $\mathrm{X} 2 / \mathrm{df}$ & $\mathrm{CFI}$ & $\mathrm{TLI}$ & $\mathrm{RMSEA}$ & SRMR \\
\hline $\begin{array}{l}\text { The girl } \\
\text { model }\end{array}$ & 5.071 & $\begin{array}{l}5.07 \\
1\end{array}$ & 5.071 & 5.071 & 5.071 \\
\hline $\begin{array}{l}\text { The boy } \\
\text { model }\end{array}$ & 4.631 & $\begin{array}{l}0.96 \\
4\end{array}$ & 0.937 & 0.074 & 0.046 \\
\hline
\end{tabular}

The structure chart of path coefficient shows that boys and girls in regular classes have significant direct positive prediction effect on self-esteem and school identity (boys: $\beta=0.364, \mathrm{P}<0.01$; Beta $=0.264, \mathrm{P}<0.01$. Female: $\beta=0.343, \mathrm{P}<0.01$; Beta $=0.168, \mathrm{P}<0.01$ ); Meanwhile, self-esteem positively predicted school identity (male $\beta=0.479, \quad \mathrm{P}<0.01$; Female $\beta=0.514$, $\mathrm{P}<0.01$ ). The results that self-esteem had a partial mediating effect on the relationship between class placement and school identity (male $=0.174, \mathrm{P}<0.01$; Female $=0.176, \mathrm{P}<0.01$ ) accounted for $39.8 \%$ in males and $51.2 \%$ in female for both male and female. This shows that the mediating effect of self-esteem exists in both male and female students, but it is larger in female students.

\section{RESEARCH CONCLUSIONS}

The results of this study show that there is a significant positive correlation between class teaching and the school identity of ordinary class students, and also has a positive predictive effect on the school identity of ordinary class students. This may be because, as an exogenous rational identity, school identity is students' identification with the influence of school culture, education, and values[15 16]. It is also the acquisition of a sense of belonging and identity built in the school and can affect students' positive emotional experience. The cognition-extension view of positive emotion holds that positive emotion can expand people's perception and way of thinking and influence students' attitude and behavior by guiding their perception and way of thinking of school culture, values, and moral norms. The other aspect, based on the theory of social identity, when students from the school identity, individuals specification acceptance, internalize school, education ideas, and values, and your performance in school, the relationship with the school into the self-concept, behavior and personality development toward the school established development goals, to enhance their sense of identity and belonging to the school.

This study also found that class placement can further influence students' school identity through self-esteem, and self-esteem plays a mediating role in the relationship between class placement and school identity. Existing research shows that teaching for students in the regular class divide into classes, not just the fairness issue of education resources, more importantly, under the environment of unfair, the regular class students self-esteem is gradually hit by the environment and external negative factors, the result is they don't agree to the existing environment to disgust, which is the direct expression on the gradual lack of identity to the school[17]. Although both male and female students' attitudes to class teaching can influence their school identity through self-esteem, the effect of this effect on female students is greater than that of male students, and the direct effect of male students' attitude to class teaching on their school identity is greater than that of female students. The developmental orientation theory of self-esteem function also argues that self-esteem can affect self-competence. The individual's sense of ability can encourage them to have the courage to face challenges and overcome difficulties, stimulate the sense of responsibility, affect the individual's social behavior and behavioral tendency, and pursue the sustainable development of self and personality[18]. Class teaching has a very negative effect on the personal growth of ordinary class students.

\section{CONCLUSION}

The fundamental aim of education is "complete human development". The complete development of human beings is the unity of knowledge, affection, and behavior, the unity of inner and outer parts, and the harmonious integration of human and nature, human and society, and human and human. The "social man" must have rich emotion, clear self-consciousness, and a sound personality. Lack of any aspect, human development is not comprehensive, incomplete. Educational equity is an important means to achieve "the complete development of human beings". The unfairness of education will tear the whole development of people. The stratified and class-divided teaching is a microscopic educational injustice, which openly disregards the equality of educational rights and fails to realize the all-around development of people.

First of all, the level of love and care constitutes the barriers of emotional communication between teachers and students, so that students become increasingly indifferent to emotion. Education is rooted in love, "there is no education without love", love is expressed through emotion, "emotion is mutually given". "Each human being exists as one an other's emotional needs and is mutually acknowledged (and accepted) when one feels the loving care of the other." The premise of educational activities is the mutual acceptance and recognition of teachers and students, which is rooted in love. Love is selfless and unconditional. Fairness and equality form the basis of love. In the hierarchical teaching organization form, education's care for students is distinguished by the level, and there is a grade 
difference in love. Hierarchical love is not easily accepted. The students who are classified into the lower level first feel the rank and discrimination, not love, their mentality is humiliating, the mood is resistant. There is estrangement in the emotional communication between teachers and students, and students' emotional indifference and inner closure are the initiators of stratified class teaching. School as the carrier of education, unfair education, and hierarchical care make ordinary class students gradually lose confidence in learning, and also lose the sense of belonging and identity to the school.

Secondly, the stratified class teaching eliminates self-esteem, inhibits the development of individual self-consciousness, and makes it difficult to form a sound personality. Sound personality is the premise for citizens to participate in social life, self-consciousness is the basis of the sound personality, and self-esteem is an important part of self-consciousness. Self-esteem comes from the equality of rights, the inequality of rights will only produce discrimination. Only when individuals are granted equal rights and recognized, can they harmoniously integrate into society and gain dignity. Society is a community of individuals, in which every individual is a subject with equal rights and needs to be respected. Only when individuals actively recognize and respect the rights of others, can the relationship of rights and obligations between subjects be established, mutual respect between subjects occur, and self-esteem occurs. In other words, self-esteem is respect for others, and self-esteem is formed by respect for others. Self-esteem is the internal driving force for individuals to achieve further development, which is based on respect for others and equality of rights. Students are subjects with equal rights to education and should be treated equally in education. In the opposite direction, stratified class teaching grants different rights to the same subjects -more rights to subjects in a socially dominant position, and fewer rights to subjects in a socially disadvantaged position. Unequal rights lead to discrimination, which is incompatible with self-esteem. In an environment without self-esteem, there is no way to identify with it, and the lack of school identity results from this.

Finally, due to the psychological and physiological influence of adolescent girls, girls at this stage are more emotionally sensitive than boys, which has a more profound impact on the self-esteem brought by class teaching, and the lack of school identity of girls is also more obvious.

Therefore, we should face up to the disadvantages and adverse effects of class teaching, constantly improve education and teaching methods, while paying attention to the cultivation of students' knowledge and skills, we should also pay attention to students' mental health.

\section{REFERENCES}

[1] Che Saiya, Bao Xiaolan. Research Review and Discussion on Hierarchical Teaching Method [J]. Education and Teaching Forum,2021,(07):153-156.

[2] [1]Liu Yu. A Study on High school Classification based on the Perspective of Sociology [J]. Science Consulting (Science \& Technology Management),2021,(02):241-242.

[3] Francis B, et al. Exploring the relative lack of impact of research on 'ability grouping' in England: a discourse analytic account[J].Cambridge Journal of Education,2017,47(1):1-17.

[4] Cheng HONGyan. Attitude Survey and Alternative Strategies on Middle School Classification [J]. Management of Primary and Secondary Schools,2009(9):18-22.

[5] Zheng Jianhong, Chen Jianling. A survey on the mental health of senior High students $[\mathrm{J}]$. Chinese Journal of Health Psychology, 2007(2):124-125.

[6] Li Tianchang. A Review of Studies on Students' School Identity [J]. Journal of South China,2018,(01):99-102+112.

[7] Gu Jibao, PENG Lijun. Analysis on the influencing factors of graduate students' organizational identity: A case study of University of Science and Technology of China [J]. Academic Degree and Graduate Education, 2011 (8): 56-62.

[8] CANO F, MARTIN A J, GINNS P, et al. Students' self-worth protection and approaches to learning in higher education: predictors and consequences $[\mathrm{J}]$.Higher Education.2018,76(1):163-181.

[9] Yin Rong, Zhang Feifei. The Mechanism of group identity in cluster behavior. Advances in psychological science, 2015,23 (9) : 1637-1647

[10] Huang Min-er. The nature of self-esteem. Journal of Guangzhou Teachers College (Social Science Edition), 1996, 2:38-43

[11] Shi Zhongying. School culture, school identity, and school development. Chinese Teachers, 2006, 12: 6-8

[12] Fang Ping, Ma Yan, Zhu Wenlong, et al. Status quo and problems of self-esteem research. Advances in Psychological Science, 2016, 24(9): 1427-1434

[13] Yin Rong, Zhang Feifei. The mechanism of group identity in cluster behavior. Advances in Psychological Science, 2015, 23(9): 1637-1646.

[14] Li Yajun, Shao Shuichao. Research on the status 
quo and promotion strategy of Junior Middle School Students' school identity. Chinese Journal of Education, 2019, 2: 98-102

[15] Ying Qiu. Tracing and analysis of developing trend and influencing factors of self-esteem of junior middle school teenagers. Acta Psychologica Sinica, 2015, 47(6): 787-796

[16] Zhang Yongxin, et al. The lifelong development of self-esteem. Advances in Psychological Science, 2010, 18(7): 1128-1135

[17] Shelling Dai, Guo Yilong. The influence of class teaching on students' mental toughness. Mental Health Education in Primary and Secondary Schools, No. 26, 2019 (Total No. 409) 4-7.

[18] Xu Shuangcheng, Zhang Li. The Ruin of Education equity: Stratified class teaching. 